\title{
Long-term complete remission under TDMI and local radiotherapy treatment on an inflammatory HER2-positive breast cancer
}

\begin{abstract}
Premenopausal female patient is diagnosed at the age of 45 for locally advanced inflammatory HER2-positive breast cancer with axillary node involvement. Her disease reveals bad prognostic factors. In spite of radical mastectomy after intensive neoadjuvant treatment based on chemotherapy and trastuzumab-pertuzumab, early skin infiltration recurrence overcomes. She receives local radiotherapy and TDM1 therapy as first advanced disease line. Toxic side effects are not relevant. She achieves four-years-long disease-free survival. Precise treatment selection is challenging but can find out cancer defeat.
\end{abstract}

Volume I2 Issue I - 202I

Miguel Borregón, Katherin Martínez, Irene Ramos, Alba Ramos, Manuel Alejandro Mazariegos, Beatriz Berzal, Cristina Gómez, Margarita Díez, Irene Otero, Juan David Cárdenas, José Ignacio Chacón, Carmen Esteban

Medical Oncology Department, Hospital Virgen de la Salud, Toledo, Spain

Correspondence: Miguel Borregón Rivilla, Medical Oncology Department, Hospital Virgen de la Salud, Toledo, Spain, Tel 680870I I7, Email miguelborregonrivilla@gmail.com

Received: December 30, 2020 | Published: January 14, 202 I

\section{Clinical case}

Premenopausal patient, with no significant medical history and good functional status is diagnosed in June 2016, at the age of 45, for breast cancer.

The patient has good family support, she lives at home with her husband and two daughters. She works in her own business. As cancer family history, her father suffered from pancreatic carcinoma at an advanced age.

The symptomatic debut is by self-examination of left breast enlargement, three months of evolution. Physical examination at diagnosis reveals diffused left breast growth, without delimitation of a clear nodule. Skin of the breast shows discrete diffuse subcutaneous oedema and discrete retraction of the nipple. Ipsilateral indurated lymph nodes are palpated. Complete body physical examination does not reveal other alterations.

Mammography (Figure 1) and mammary ultrasound show left mammary pattern of patchy glandular type b densities, with inflammatory signs, skin thickening and some nodular lesions, being characterised as grade 4 breast-image-reporting-and-data-system (BIRADS) lesion.

Magnetic resonance imaging (MRI) of her breast (Figures 2-4), detects diffuse pathological changes in both upper quadrants of her left breast, with skin involvement at the anterior level of areolanipple complex, approximately $90 \times 80 \times 65 \mathrm{~mm}$. There is no objective affectation of homolateral anterior pectoral muscle. Suspicious adenopathy is detected in left axillary area. It is categorised as grade 5 BIRADS lesion.

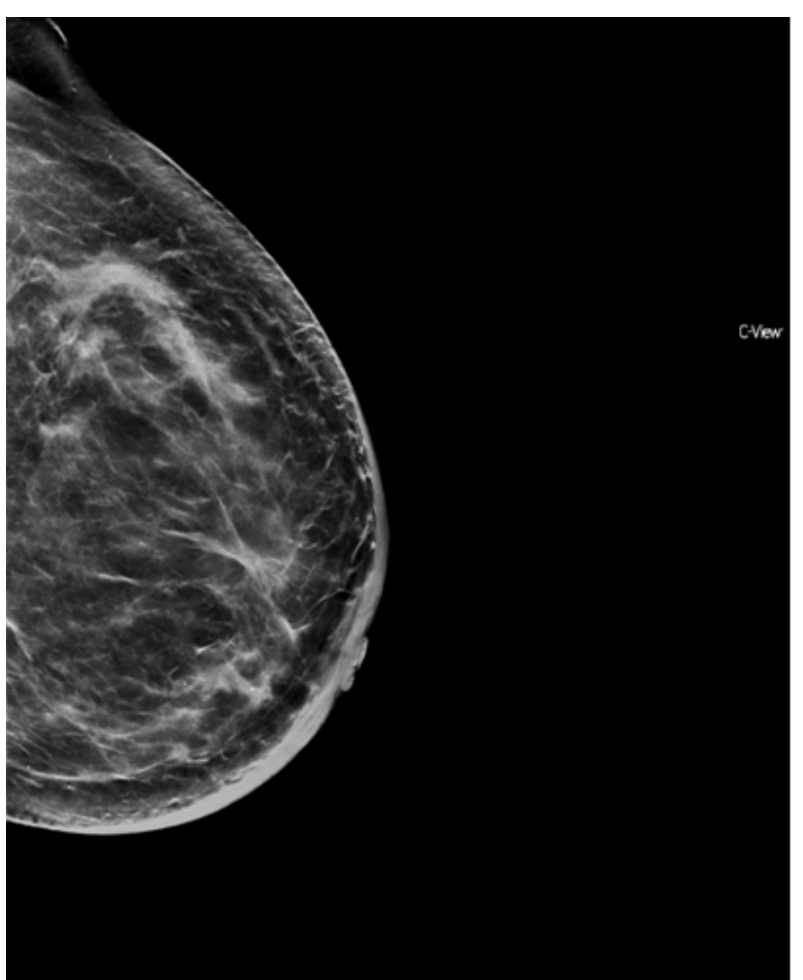

Figure I Mammography and mammary ultrasound show a left mammary pattern of patchy glandular type b densities, with inflammatory signs, skin thickening and some nodular lesions. 


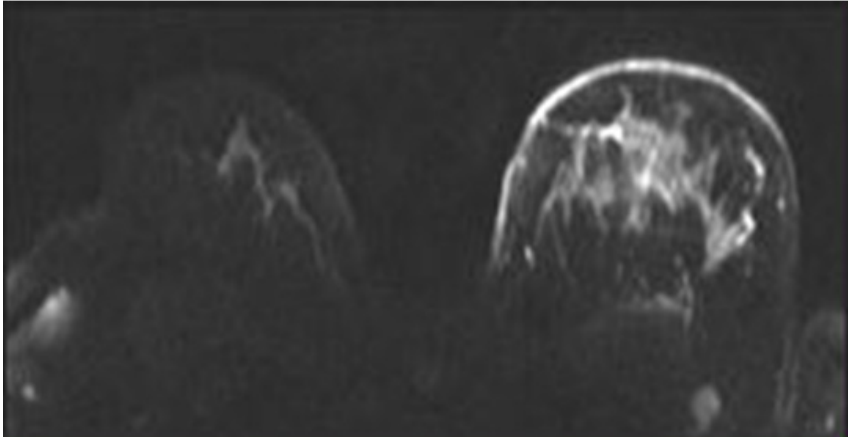

Figure 2 Magnetic resonance imaging (MRI) of the breast.

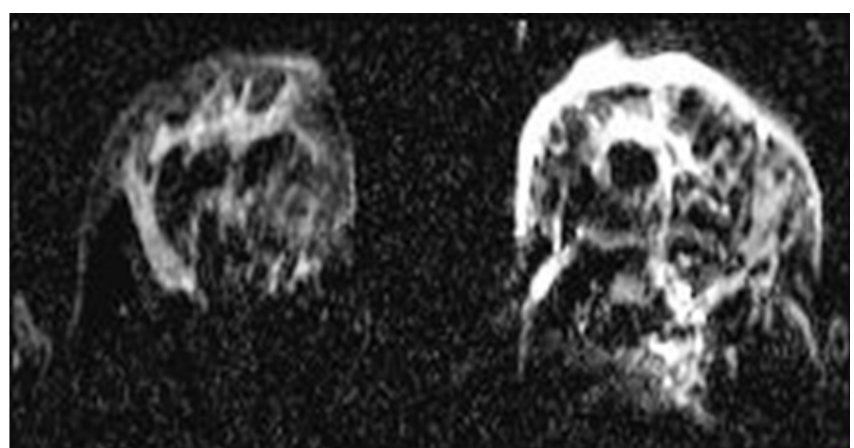

Figure 3 Magnetic resonance imaging (MRI) of the breast, detects diffuse pathological changes in both upper quadrants of the left breast.

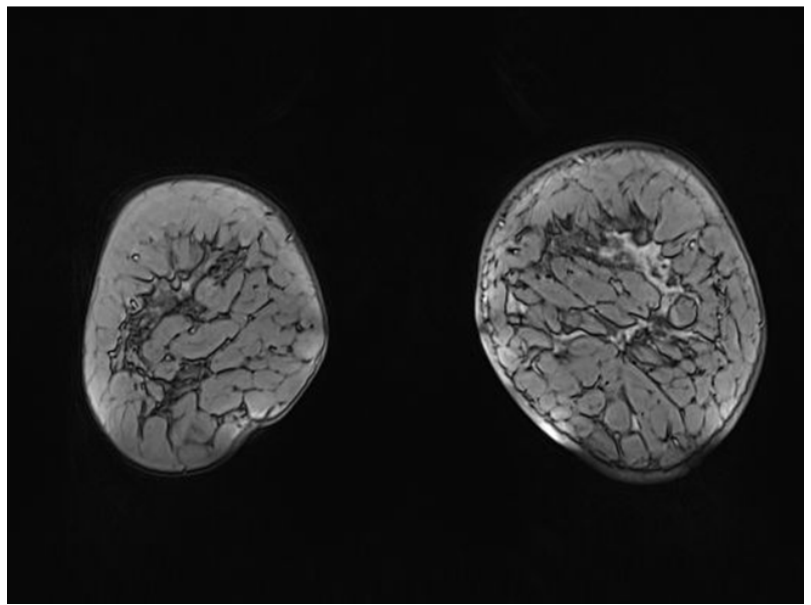

Figure 4 Magnetic resonance imaging (MRI) of the breast with skin involvement at the anterior level and of the areola-nipple complex, of approximately $90 \times 80 \times 65 \mathrm{~mm}$.

Two core needle biopsies are performed, one at the upper external quadrant and the other at the lower quadrant junction, leaving a marker in the area. They reveal ductal infiltrating carcinoma histology, with positive cytokeratin 19 (CK19), positive e-cadherin and negative $\mathrm{p} 53$. Histological grade is Nottingham grade 3 , with apocrine changes and extensive desmoplastic stroma. Immunohistochemistry (IHQ) reveals negative estrogen (ER) and progesterone (RP) receptors and human epidermal growth factor (HER2) receptor 2 overexpression (three crosses), thus a HER2-enriched subtype. Cell proliferation marker Ki67 is $25 \%$. Biopsy from axillary adenopathy confirms malignant histology.

Clinical stage at diagnosis is locally advanced inflammatory cancer, without clear anatomically delimitation, with homolateral axillary node involvement, stage IIIB (cT4d, cN1-2, cM0) from the TNM $8^{\text {th }}$ edition staging system of the American Joint Committee on Cancer (AJCC).

The patient is referred to Medical Oncology for extension study and assessment of neoadjuvant treatment. Complete blood test, body computed tomography (CT) scan and skeletal scintigraphy do not show any distant findings.

Neoadjuvant chemotherapy treatment is administered between June and October 2016. She receives four intravenous cycles of cyclophosphamide $600 \mathrm{mg} / \mathrm{m}^{2}+$ epirubicin $90 \mathrm{mg} / \mathrm{m}^{2}$ every two weeks. Main toxicities are grade 2 nausea, grade 1 anaemia, grade 1 oral mucositis, grade 1 fatigue and mild telorrhoea.

First clinical revaluation does not reveal important improvement. Diffuse breast induration and rigid axillary adenopathy have hardly decreased. Skin remains irregularly erythematous. Control mammography is categorized as stable disease, with no important radiological changes.

Second course of neoadjuvant treatment based on four intravenous cycles of docetaxel $75 \mathrm{mg} / \mathrm{m}^{2}+$ trastuzumab $6 \mathrm{mg} / \mathrm{kg}+$ pertuzumab $420 \mathrm{mg}$ every three weeks is given. Main toxicities are grade 2 oral mucositis, grade 2 anaemia, grade 2 neutropenia and moderate mixed anxiety-depressive disorder, which requires anxiolytic treatment. She precises $10 \%$ docetaxel dose reduction and colony-stimulating-factor and erythropoietin support.

Once neoadjuvant treatment is finished, and with a poor clinical and radiological response (stable disease on objective radiological measures), the patient is referred to general surgery. She is operated in December 2016 by modified radical mastectomy including skin involvement and Berg levels I-II-III axillary dissection.

Pathological study of the surgical specimen reveals a mammary parenchyma with the presence of a tumour bed of approximately $100 \times 70 \mathrm{~mm}$, made up of $90 \%$ residual tumour cellularity, corresponding to extensive high nuclear grade intraductal carcinoma with necrosis and central microcalcifications. The remaining $10 \%$ of the tumour corresponds to multifocal and multicentric infiltrating ductal carcinoma. Extensive lympho-vascular and dermal lymph vessel invasion is identified. Skin and nipple are infiltrated. Surgical borders are not infiltrated. Left axillary lymphadenectomy reveals metastases in five of seven isolated lymph nodes, with extra-nodal invasion and maximum diameter of $12 \mathrm{~mm}$. Residual tumour burden is 3,817 (MD Anderson Class III, extensive residual disease). Pathological stage is pT4, pN2, pMx.

On her first medical visit, one month after surgery, on January 2017, the patient presents local skin alterations that lead to the suspicion of disease recurrence/persistence. Diffuse and difficult to delimit infiltrative aspect erythema on the skin on both sides of the scar has showed up. Skin biopsy is compatible with carcinoma infiltration, with HER2-positive histology and Ki67 of 40\%. Tumour marker CA-15.3 is within normal limits $(26,1 \mathrm{U} / \mathrm{ml})$.

Body CT scan and skeletal scintigraphy do not find distant disease. It is categorized and diagnosed as stage IV breast cancer due to local skin recurrence. Since it is a HER2-positive tumour, and it has exhibited low sensitivity to trastuzumab-pertuzumab, it is decided to start systemic treatment with a different drug: Ado-trastuzumabemtansine (TDM1), at a dose of intravenous $3,6 \mathrm{mg} / \mathrm{kg}$ every three weeks. The patient presents normal heart function, corroborated by electrocardiogram and echocardiogram tests. 
TDM1 is well tolerated. Main toxicities are grade 1 myalgia, grade 1 hepatitis, grade 1 anaemia, grade 2 fatigue, and grade 2 emesis.

Based on the restricted cutaneous affectation (oligometastatic disease scenario), and the inflammatory carcinoma presentation, it is decided to provide complementary treatment with local radiotherapy in curative intent. 64 grays (Gy) dose divided into 30 sessions of 215 centi-grays (cGy) is applied. Main toxicity is grade 2 acute radiodermatitis, with subsequent mild-moderate skin fibrosis, requiring opioid analgesics.

Once radiotherapy is finished and eight TDM1 cycles have been administered, cutaneous alteration completely disappears. Body CT scan does not find distance disease. Echocardiogram rules out cardiac toxicity. It is catalogued as complete clinical response and it is decided to continue systemic therapy based on TDM1 until disease progression or unacceptable toxicity.

Protocol blood tests and image studies every three months by body CT scan, annual skeletal scintigraphy and positron emission tomography (PET) scan performed on one occasion, rule out tumour relapse. Tumour markers are within normal range. On December 2020, after 46 TDM1 cycles, the patient exhibits 4 years complete remission.

Main complications during the course of her cancer treatment and follow-uparemild leftbasalpneumoniarelated toradiation pneumonitis; depressive symptoms and insomnia, assessed by psychiatry; and mild coronavirus infection in October 2020, symptoms consisting of fever, headache, ageusia and anosmia, not requiring hospital admission. She also presents recurrent cumulative haematological toxicity related to TDM1, needing occasionally erythropoietin, colony stimulating factor and TDM1 short discontinuation.

In summary, combination of TDM1 and local radiotherapy become a successful systemic therapy, with an extended disease-free survival together with a manageable toxic profile and good quality of life.

\section{Discussion}

An inflammatory HER2-positive tumour diagnosed as locally advanced tumour with lymph node and skin involvement has been exposed. Clinical and molecular profile reveal a high relapse risk. Selecting the best treatment option in this particular setting is a challenge. Patient's survival and symptoms depend on this selection. Systemic therapy based on TDM1 becomes an effective and successful therapy. We are providing review of the most important points of the clinical case. ${ }^{1,2}$

Inflammatory breast cancer represents only $1-5 \%$ of breast cancers in the United states. It is characterized by diffuse erythema and oedema ("peau d'orange") of the breast often without an underlying palpable mass. It typically has a rapid onset and is often initially mistaken as infection and treated with antibiotics before the diagnosis is established. Pathogenesis results from tumour emboli in the dermal lymphatics. It is more likely to be high-grade, HER2 overexpressing and lacking in hormone receptor expression compared with other presentations of breast cancer. It is related with substantial risk of metastatic disease and higher risk of recurrence. It should be routinely treated with postmastectomy radiotherapy, despite a pathologic complete response to neoadjuvant chemotherapy. In spite of modern multimodality therapy, approximately $20 \%$ of these tumours will experience local-regional recurrence. This kind of recurrence must be treated according to metastatic breast cancer guidelines. ${ }^{3,4}$
Approximately $20 \%$ of all breast cancers present gene amplification or overexpression of human epidermal growth factor receptor 2 (HER2), resulting in a more aggressive phenotype and a poor prognosis. Trastuzumab is the first line therapy both in adjuvant and advanced disease setting. Some patients will present primary or acquired resistance to trastuzumab. In this setting, TDM1 emerges as a rational alternative. ${ }^{5,6}$

TDM1 is an antibody-drug-conjugate that is made up of trastuzumab and the small-molecule microtubule inhibitor DM1. Upon binding to HER2 receptor, TDM1 undergoes receptor-mediated internalization and lysosomal degradation, leading to intracellular release of the DM1 molecule. Binding of DM1 to tubulin leads to disruption of the microtubule network, resulting in cell-cycle arrest and apoptosis. It also inhibits HER2 downstream signalling pathways and develops immunologic-mediated mechanisms. It has demonstrated important prolonged progression-free and overall survival and acceptable toxicity profile in HER2-positive advanced breast cancer previously treated with trastuzumab and a taxane, receiving American food and drug administration (FDA) approval at this context. ${ }^{7,8}$

This clinical case illustrates a successful tumour management. Finding out the best treatment option for each singular patient constitutes a difficult challenge. Deep study of tumour scenario by approaching patient clinical characteristics, molecular profile, so as to recurrence risk and prior therapies responses, allow oncologists to select successful therapies which can defeat cancer.

\section{Conclusion}

Treatment goals on patients with advanced breast cancer include life prolongation, tumour burden control, reduction in cancer-related symptoms and complications, and maintenance of quality of life. Therapy is not generally considered curative. The patient we present symbolizes a faithful example of these main points and an open window to advance disease total recovery. 4-years-complete-response and an acceptable tolerance profile are achieved with TDM1 and local radiotherapy, revealing their therapeutic potential on aggressive HER2-positive breast cancer setting.

\section{Acknowledgments}

The authors of this manuscript would like to be grateful to the patient whose clinical case has been exposed. Her help to the contribution of medical science development needs to be mentioned and acclaimed.

We would like to thank Dr. Carmen Esteban, Medical Oncologist of Medical Oncology Department in Hospital Virgen de la Salud from Toledo, for her contribution to the education of new oncologist residents. She has always been open to help and guide clinical and researching projects and she is the light that leads this report and many other researching activities.

\section{Funding}

There is no other funding source than the Spanish Government basic salary for medical doctors. No external private firm has participated on this research.

\section{Conflicts of interest}

All authors from this manuscript declare that there is no conflict of interest on its publication. Its main aim and motivation are helping to medical science development. 


\section{References}

1. Kobayashi K, Ito Y, Matsuura M, et al. Impact of immunohistological subtypes on the long-term prognosis of patients with metastatic breast cancer. Surg Today. 2016;46(7):821-826.

2. Malmgren JA, Mayer M, Atwood MK, et al. Differential presentation and survival of de novo and recurrent metastatic breast cancer over time: 1990-2010. Breast Cancer Res Treat. 2018;167(2):579-590.

3. Siegel RL, Miller KD, Jemal A. Cancer statistics, 2019. CA Cancer J Clin. 2019;69:7.

4. Colleoni M, Sun Z, Price KN, et al. Annual hazard rates of recurrence for breast cancer during 24 years of follow-up: results from the International breast cancer study group trials I to V. J Clin Oncol. 2016;34:927.

5. Slamon DJ, Clark GM, Wong SG, et al. Human breast cancer: correlation of relapse and survival with amplification of the HER-2/neu oncogene. Science. 1987;235:177-182.
6. Ross JS, Slodkowska EA, Symmans WF, et al. The HER-2 receptor and breast cancer: ten years of targeted anti-HER-2 therapy and personalized medicine. Oncologist. 2009;14:320-368.

7. Junttila TT, Li G, Parsons K, et al. Trastuzumab-DM1 (T-DM1) retains all the mechanisms of action of trastuzumab and efficiently inhibits growth of lapatinib insensitive breast cancer. Breast Cancer Res Treat. 2011; 128:347-356.

8. Diéras V, Miles D, Verma $\mathrm{S}$, et al. Trastuzumab emtansine versus capecitabine plus lapatinib in patients with previously treated HER2positive advanced breast cancer (EMILIA): a descriptive analysis of final overall survival results from a randomised, open-label, phase 3 trial. Lancet Oncol. 2017;18(6):732-742. 